\title{
Affinity studies of hypocrellin B and mono-cysteine substituted hypocrellin B with CT-DNA using spectroscopic methods
}

\author{
Shaohua Wei ${ }^{\text {a,b,* }}$, Jiahong Zhou ${ }^{\text {b }}$, Yuying Feng ${ }^{\text {b }}$, Deyin Huang ${ }^{\text {a }}$, Xuesong Wang ${ }^{\text {b,* }}$, \\ Baowen Zhang ${ }^{\mathrm{c}, *}$ and Jian Shen ${ }^{\mathrm{b}}$ \\ a School of Chemistry and Chemical Technology, Shanghai JiaoTong University, Shanghai 200240, \\ PR China \\ ${ }^{\mathrm{b}}$ Jiangsu Engineering Research Center for Bio-medical Function Material, Nanjing Normal \\ University, Nanjing 210097, PR China \\ ${ }^{\mathrm{c}}$ Technical Institute of Physics and Chemistry, Chinese Academy of Sciences, Beijing 100101, \\ PR China
}

Abstract. The interaction of anticancer drug hypocrellin B (HB) and mono-cysteine substituted hypocrellin B (MCHB) with calf thymus deoxyribonucleic acid (CT-DNA) has been investigated using spectral methods. The results of UV-visible spectra showed that the HB and MCHB can intercalate into the base-stacking domain of the CT-DNA double helix. Further studies based on fluorescence spectroscopy and circular dichroism (CD) spectroscopy also supported the intercalation mechanism.

Keywords: Hypocrellin B, MCHB, CT-DNA, intercalation mechanism, circular dichroism spectroscopy, fluorescence spectroscopy, anticancer drugs

\section{Introduction}

Hypocrellin B (abbreviated as HB) (Fig. 1) has been isolated from natural fungus sacs of Hypocrella bambusae [1] in China. This lipid-soluble perylenequinonoid derivative has been tested as a phototherapeutic agent in medicines to cure certain skin diseases. Recently, HB was also found to display antitumoral activities [2,3]. HB and its derivatives exhibit photosensitized damage to DNA. However, the details of the mechanism of this process are poorly understood due to the low water solubility of $\mathrm{HB}$.

Studies investigating the therapeutic mechanism and photochemistry of HB have been carried out in systems such as liposomes [4] or aqueous micelles to overcome the problem of its low water solubility. However, under such conditions the HB molecule cannot interact with DNA directly. Therefore, an understanding of the precise nature of the interaction between HB molecule and DNA molecule remains unclear. An answer to this question is also vital for understanding the mechanism of anticancer effect of this drug molecule. However, up to now, no experiments about this interaction were reported. The aim

\footnotetext{
*Corresponding authors. Tel.: +86 258359 8253; Fax: +86 258359 8250; E-mail: shwei@ njnu.edu.cn.
} 


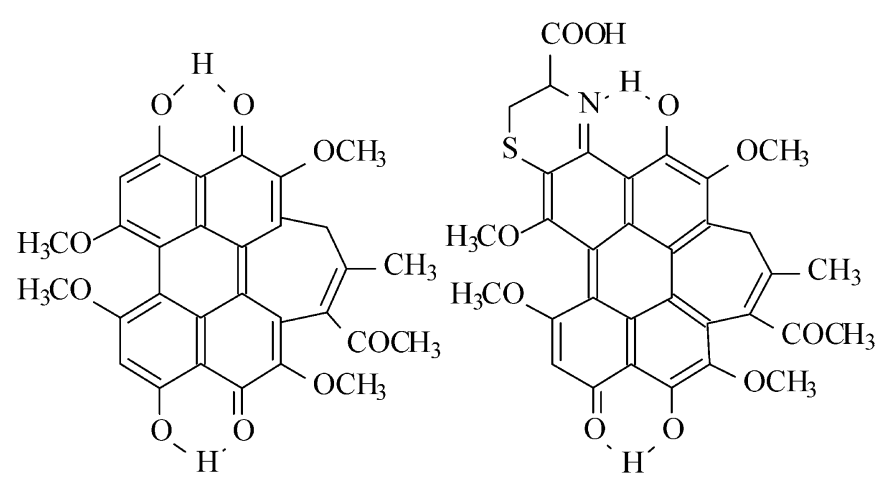

Fig. 1. Structure of HB (left) and MCHB (right).

of the current study to address this issue by investigating the mechanism of interaction between $\mathrm{HB}$ and MCHB with CT-DNA using a range of spectroscopic techniques. The results, which were obtained from UV-visible spectra, fluorescence spectra and circular dichroism spectra, indicated that $\mathrm{HB}$ and MCHB could intercalate into the base pairs of CT-DNA molecule.

\section{Experimental}

\subsection{Materials}

The Calf thymus DNA (CT-DNA) and Ethidium bromide (EB) (2,7-diamino-9-phenyl-10-ethylphenanthridinium bromide) were obtained from Huamei Chemical Co. (Beijing), and used without further purification. CT-DNA concentrations were determined spectrophotometrically with an extinction coefficient of $6600 \mathrm{~mol}^{-1} \mathrm{~cm}^{-1}$ at $260 \mathrm{~nm}$, and the stock solutions of CT-DNA was prepared in double distilled water and stored in a freezer when not in use.

HB was prepared according to the literature [6]. Dimethylsulfoxide (DMSO) as the solvent was dried by distillation over $\mathrm{KOH}$ prior to use. The solutions of $\mathrm{HB}$ were prepared by adding small amounts of concentrated DMSO solutions of HB to double distilled water, the final percentage of DMSO in the solution was never above $0.15 \mathrm{mM}$.

All experimental solutions were buffered at $\mathrm{pH} 7.1$ by $1.0 \mathrm{mM} \mathrm{PBS}$, and $10 \mathrm{mM} \mathrm{NaCl}$ was added to keep up the native conformation of CT-DNA.

\subsection{Spectral measurements}

A Shimadzu Spectrophotometer UV-1601PC and an Jasco spectrometer 500-C were used for recording the absorption and circular dichroism spectra, respectively. All the mixing solutions were monitored after 24 hours in dark.

Fluorescence spectra were recorded with a Hitachi fluorescence spectrophotometer, model f- 4500 , using quartz cuvettes of path length $1.00 \mathrm{~cm}$. All the fluorescence spectra were recorded with the same ordinate, and excitation and emission slits were $5 \mathrm{~nm}$ of each. Spectra were taken immediately after mixing for 40 minutes in dark.

All spectra were measured at room temperature $\left(25^{\circ} \mathrm{C}\right)$. 


\section{Results and discussion}

\subsection{UV-visible spectra}

The UV-visible absorption spectrum changed by HB in the presence of varying amounts of CT-DNA had been shown in Fig. 2. In the region of $360 \mathrm{~nm}$ to $800 \mathrm{~nm}$, HB had a characteristic absorbance band at $466 \mathrm{~nm}$ due to $\pi-\pi^{*}$ transition (curve a) [7]. Upon addition of increasing amounts of CT-DNA to the solution containing a fixed concentration of $\mathrm{HB}(10 \mu \mathrm{M})$, notable changes in the absorption spectrum of HB were observed. The absorption bands at $466 \mathrm{~nm}$ shifted to longer wavelength, at the same time, the spectrum showed strong decreases in the peak intensities (hypochromicity) on increasing the concentration of CT-DNA. When $120 \mu \mathrm{l}$ of CT-DNA was added, the peak at $466 \mathrm{~nm}$ shifted to $535 \mathrm{~nm}$ (curve b), and the intensity decreased 34\%. It is known that the probes, which stack with DNA, are planar; and planarity is an important criterion for effective intercalation into the helix. Changes in the absorption band (hypochromism) and shift in the wavelength (shift-red) are a hallmark for intercalation of drugs to DNA helix [8]. Therefore, such pronounced hypochromism and bathochromism observed from the absorption spectrum of HB were suggested to be due to a strong intercalation of HB molecule into CT-DNA base pairs. This implied a close proximity of the HB chromophore to the CT-DNA base pairs, i.e., a strong overlap between the electronic states of the intercalating chromophore and that of the CT-DNA bases occurred.

In contrast to the above results on $\mathrm{HB}, \mathrm{MCHB}$ had two characteristic absorbance bands at 591 and $753 \mathrm{~nm}$ within the region of $400 \mathrm{~nm}$ to $800 \mathrm{~nm}$ [9] (curve a in Fig. 3). When CT-DNA was added into the solution of MCHB, two absorption bands shift to longer wavelength and the peak intensities also strongly decrease. After $120 \mu \mathrm{l}$ of CT-DNA was added, the absorption band at $591 \mathrm{~nm}$ shifted to 606 (curve $\mathrm{b}$ in Fig. 3), and the intensities decreased by $24 \%$. Thus, the binding intensity of HB to CTDNA was larger than MCHB. The reason might be that the space conformation of MCHB decreases its intercalating ability.

\subsection{Fluorescence spectra}

The binding of HB to CT-DNA was also studied by fluorescence spectroscopy. Figure 4 shows the emission spectra of HB in the absence and presence of CT-DNA. When HB was excited at $480 \mathrm{~nm}$, it

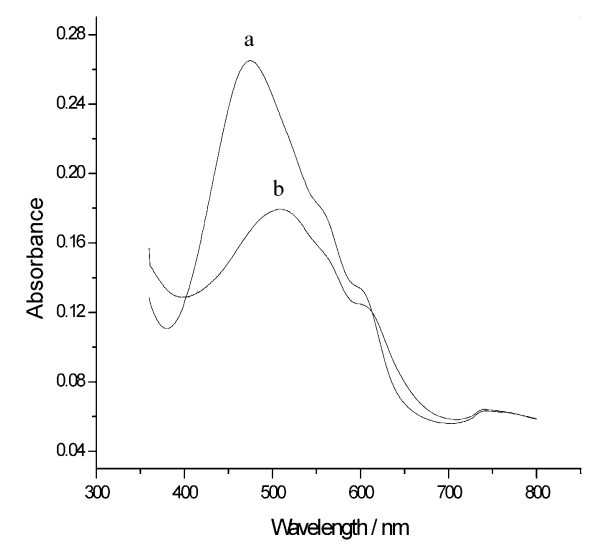

Fig. 2. UV-visible absorption spectrum of HB in the absence (a) and presence (b) of CT-DNA. 


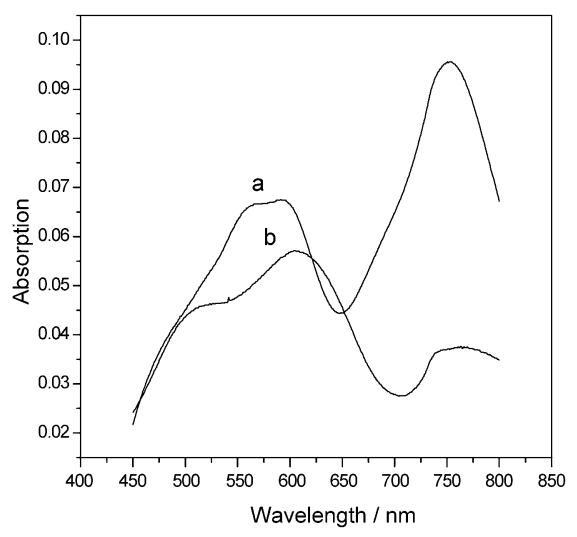

Fig. 3. UV-visible absorption spectrum of MCHB in the absence (a) and presence (b) of CT-DNA.

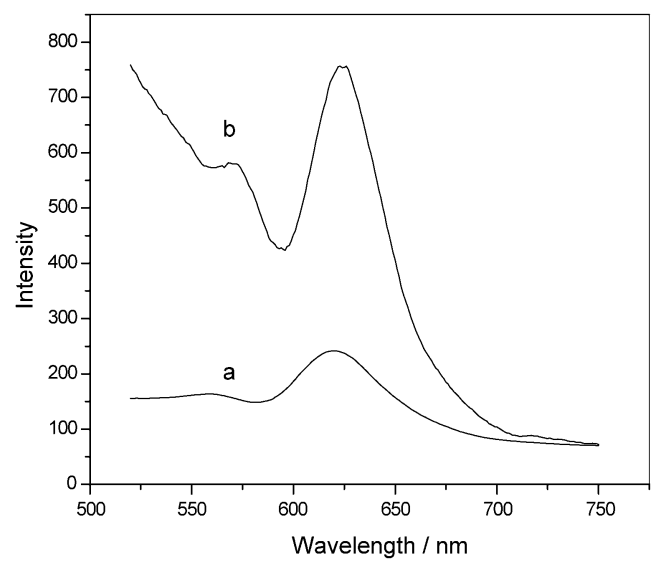

Fig. 4. Emission spectra of HB in the absence (a) and presence (b) of CT-DNA.

had a weak fluoresces peak around $619 \mathrm{~nm}$ (Fig. 4, curve a). Addition of CT-DNA caused a gradual increase in the fluorescence intensity and emission maximum also shifted by $7 \mathrm{~nm}$ to longer wavelength $(626 \mathrm{~nm})$ (Fig. 4, curve b). This effect arose, because in the presence of CT-DNA the HB molecules were bound in a relatively non-polar environment compared to water; and the collisional frequency of the solvent molecules with the HB molecules whose planar aromatic group had stacked between adjacent base pairs of CT-DNA decreased. The binding of HB to CT-DNA leading to a marked increase in emission intensity and a weak shift to longer wavelength also coincided with the observations for other intercalators [10]. MCHB also had a weak fluoresces peak at $620 \mathrm{~nm}$, when it was excited at $570 \mathrm{~nm}$. After the addition of CT-DNA, the similar phenomena of the gradual increase in the fluorescence intensity were also observed.

To further confirm the binding interactions, experiments with ethidium bromide fluorescence displacement were carried out. EB is the most widely used fluorescence probe for DNA structure and has recently been employed in the examination of the mode and process of drug binding to DNA. In this work, EB was also employed as a probe. The experiment was carried out in 2-ml solution of $200 \mu \mathrm{g} / \mathrm{ml} \mathrm{EB}, 100 \mu \mathrm{g} / \mathrm{ml}$ CT-DNA which was titrated with $0.83 \times 10^{-3} \mathrm{~mol} / \mathrm{l} \mathrm{HB}$ solution, and then excited at $520 \mathrm{~nm}$. Figure 5 shows the emission spectra of the CT-DNA-EB system in the presence of HB. The emission intensity 


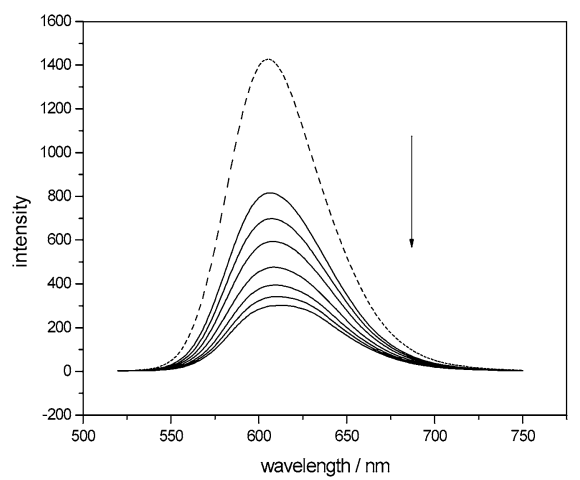

Fig. 5. Emission spectra of CT-DNA-EB system in the absence (dotted line) and presence (solid line) of increasing amounts of $0.83 \times 10^{-3} \mathrm{~mol} / \mathrm{l} \mathrm{HB}(20 \mu \mathrm{L}$ per scan $)$.

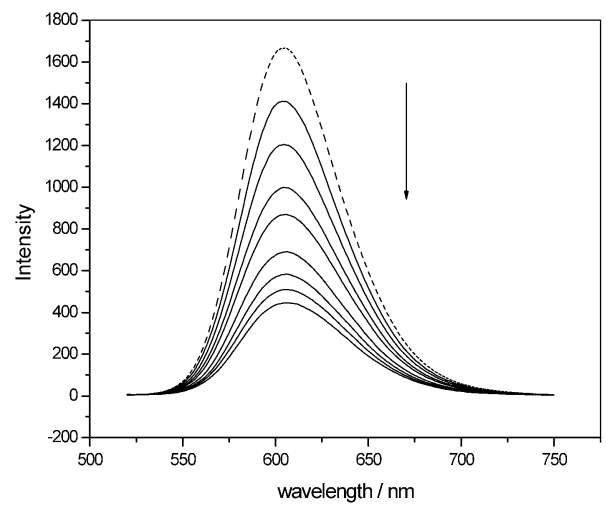

Fig. 6. Emission spectra of CT-DNA-EB system in the absence (dotted line) and presence (solid line) of increasing amounts of $0.83 \times 10^{-3} \mathrm{~mol} / \mathrm{l} \mathrm{MCHB}(20 \mu \mathrm{l}$ per scan $)$.

of the CT-DNA-EB system decreased with the increasing of the concentration of HB; and the spectra of CT-DNA-EB system revealed a small shift to longer wavelengths with HB added. The changes observed here are often as the characteristics of intercalation. This phenomenon indicated that HB replaced EB from the CT-DNA-EB system leading to the emission intensity decreasing of the CT-DNA-EB system. The above-mentioned shift was caused by an EB transferring from a hydrophobic environment into the water solution. The same experiments were done by using MCHB, and the changes of emission spectra of the CT-DNA-EB system in the presence of MCHB were shown in Fig. 6. The emission intensity of the CT-DNA-EB system also decreased as the concentration of MCHB increased, but the decreased intensity was smaller than HB. This was consistent with the results of UV-visible spectra, and indicated that the binding intensity of HB to CT-DNA was larger than MCHB.

According to the classical Stern-Volmer equation:

$$
F_{0} / F=1+\kappa_{\mathrm{q}}[Q],
$$

where $F_{0} / F$ represents the ratio of emission intensity in the absence and presence of quencher, $\kappa_{\mathrm{q}}$ is the quenching constant, $[Q]$ is the concentration of quencher. Static or dynamic quenching process plots of $F_{0} / F$ versus $[Q]$ appear linear and $\kappa_{\mathrm{q}}$ depends on temperature. The shape of the Stern-Volmer plot 


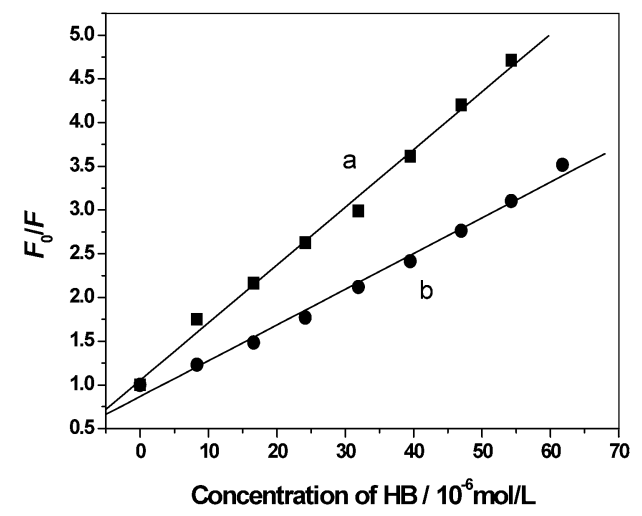

Fig. 7. Emission quenching of HB (curve a) and MCHB (curve b) to CT-DNA-EB system.

can be used to characterize the quenching as being predominantly dynamic or static. Calculating from Figs 5 and 6, the emission quenching of HB (curve a) and MCHB (curve b) to CT-DNA-EB system were shown in Fig. 7. Plotting $F_{0} / F$ versus $[\mathrm{HB}]$ or $[\mathrm{MCHB}]$ gave a straight line with linear correlation coefficient of 0.997 and 0.984 respectively. From the slope of the fitting line, the quenching constant $\left(\kappa_{\mathrm{q}}\right)$ could be calculated, then the values of $\mathrm{HB}$ and MCHB were 0.06677 and 0.0452 , respectively. These changes may suggest that only one kind of quenching process was involved, and that HB bound to CTDNA mainly by one mode, i.e., intercalation; the other weak binding modes were caused by hydrogen bond, hydrophobic action, and static action, such as groove binding and static binding [11]. Comparing the molecular structures of $\mathrm{HB}$ and $\mathrm{MCHB}$, as well as results of UV-visible spectra and fluorescence spectroscopy, the binding between MCHB and CT-DNA has another interacted binding that might be a weak groove binding.

\subsection{Circular dichroism}

Circular dichroism spectra of DNA molecules is one of the most preferable methods to monitor the conformational changes, brought about by the interacting host molecules [12]. The circular dichroism of a nucleic acid is caused by the interaction of the transition dipoles in the component of the helical structure. In Fig. 8, the changes in the circular dichroism spectrum of CT-DNA in presence of increasing concentrations of HB or MCHB were depicted, monitored from 225 to $300 \mathrm{~nm}$. Curve a was the circular dichroism spectrum of CT-DNA whose concentration was $60 \mu \mathrm{g} / \mathrm{ml}$. This exhibits a positive absorption band at $275 \mathrm{~nm}$ due to the base stacking and a negative band at $245 \mathrm{~nm}$ due to the helicity of B-DNA (curve a). When HB was added, the spectral changes consisted mainly of a significant decreasing in the intensity both of the positive band and the negative band, and the positive band shift to $280 \mathrm{~nm}$, while the negative band has no distinct shift (curve c). It is suggested that the helicity and the extent of stacking among DNA base pairs have been disturbed due to the intercalated binding between HB molecules and the base pairs of CT-DNA. From curve b, it was found that the effect of MCHB on the helicity and the extent of stacking among DNA base pairs were smaller at the same condition. Therefore, the phenomena in the circular dichroism spectrum CT-DNA in presence of increasing concentrations of HB or MCHB were coincident with the above results.

It has been reported, that HB molecular has three pKa $(-0.3,8.4$ and 11.0) [13], on the other hands, the CT-DNA molecular can hold its conformation in the $\mathrm{pH}$ range of 6.0 to 10.0. Therefore, we studied the affection of $\mathrm{HB}$ to the CD spectroscopy of CT-DNA at three $\mathrm{pH}$ conditions (6.0, 7.0 and 9.0). The 


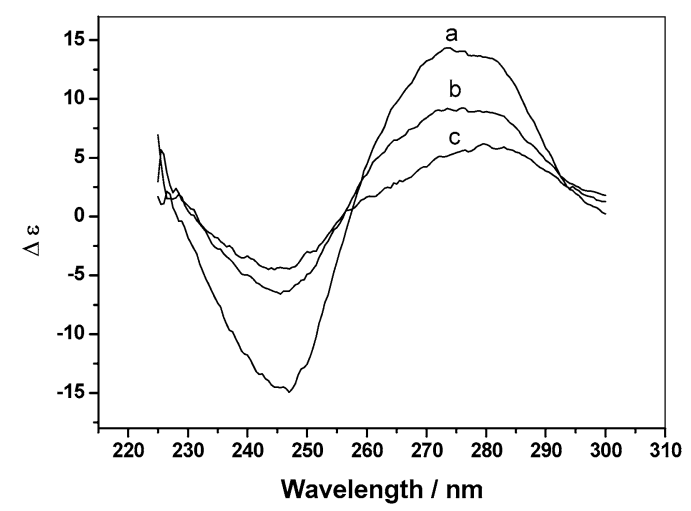

Fig. 8. The CD spectra changes of CT-DNA by addition of HB or MCHB. a: CT-DNA; b: MCHB + CT-DNA; c: HB + CT-DNA.

results suggested that the intensities of the negative peaks have no obvious change at three $\mathrm{pH}$ values, but the intensities of positive peaks weakened with the $\mathrm{pH}$ value of the solution decreasing. It indicated that the $\mathrm{pH}$ changes of the solution cannot change the ellipticity of CT-DNA in the process of interaction between HB and CT-DNA, but affect the base stacking.

\subsection{Melting temperature $\left(T_{\mathrm{m}}\right)$ of CT-DNA}

The melting temperature $\left(T_{\mathrm{m}}\right)$, at which the double helical deforms into single stranded DNA, is a useful parameter in analyzing the mode of interaction. To understand the interaction between HB or MCHB and CT-DNA, the melting temperature $\left(T_{\mathrm{m}}\right)$ experiments were performed.

According to the results, the denaturation temperature $\left(T_{\mathrm{m}}\right)$ of CT-DNA $(40 \mu \mathrm{M})$ in $10 \mathrm{mM}$ sodium phosphate buffer $(\mathrm{pH}=7.4)$ could be calculated as $60.1^{\circ} \mathrm{C}$. When $10 \mu \mathrm{M}$ of $\mathrm{HB}$ or MCHB was added, the $T_{\mathrm{m}}$ of CT-DNA decreased to $57.2^{\circ} \mathrm{C}$ or $59.3^{\circ} \mathrm{C}$. These results also indicated that the binding intensity of HB to CT-DNA was larger than that of MCHB.

\section{Conclusion}

HB is a kind of anticancer drug whose interaction, directly, with DNA has not been previously reported although this important for understanding its mechanism of anti-cancer activity. In this paper, we have investigated the mechanism of interaction between HB or MCHB with CT-DNA using several different spectroscopic methods. It was found, when HB or MCHB interacted with CT-DNA, the characteristic absorbance bands of $\mathrm{HB}$ and MCHB displays pronounced hypochromism and bathochromism. This indicated that the HB and MCHB could intercalate into the base pairs of CT-DNA molecule in a mixed system of DMSO and water. To further confirm this binding interactions, fluorescence spectroscopy experiments, ethidium bromide fluorescence displacement experiments and circular dichroism experiments were done. All the results supported the interacted mechanism obtained from the UV-visible spectra. 


\section{Acknowledgements}

The authors would like to thank Natural Science Foundation of Jiangsu Education Department (Grant No. 03KJB150059 and 04KJB150068) for the support.

\section{References}

[1] X.Y. Wan and Y.T. Chen, A new medicine for photochemical therapy - hypocrellin A, Kexue Tongbao 24 (1980), 11481149.

[2] G.G. Miller, K. Brown, M. Ballangrud, O. Barajas, Z. Xiao, J. Tulip, J.W. Lown, J.M. Leithoff, M.J. Allalunis, R.D. Methta and R.B. Moore, Preclinical assessment of hypocrellin B and hypocrellin B derivatives for photodynamic therapy of cancer: progress update, Photochem. Photobiol. 65 (1997), 714-722.

[3] J.B. Hudson, J. Zhou, L. Harris, L. Yip and G.H.N. Towers, Hypocrellin from Hypocrella bamboosae, is phototoxic to human immunodeficiency virus, Photochem. Photobiol. 60 (1994), 253-255.

[4] W. Zou, J.Y. An and L.J. Jiang, Damage to pBR322 DNA photosensitized by hypocrellin A in liposomes and its derivatives in solution, J. Photochem. Photobiol. 33 (1996), 73-78.

[5] S. Dumas, P. Jardon, J.-C. Lepretre and A. Jeunet, Electrochemistry behaviour of hypocrellin A in acetonitrile and aqueous micellar media, New Journal Chemistry 25 (2001), 1313-1318.

[6] K.H. Zhao and L.J. Jiang, Conversion of hypocrellin A in alkaline and neutral media, Chinese Journal of Organic Chemistry 9 (1989), 252-254.

[7] J.H. Zhou, S.Q. Xia, X.S. Wang and B.W. Zhang, The photodynamic property improvement of hypocrellin A by chelation with lanthanum ions, Chem. Commu. (2003), 1372-1373.

[8] V.A. Bloomfield, D.M. Crothers and I. Tinoco, eds, Physical Chemistry of Nucleic Acids, Harper, Row, New York, 1974, p. 432.

[9] Y.Y. He, J.Y. An and L.J. Jiang, Synthesis of a new water-soluble phototherapeutic sensitizer from hypocrellin B with enhanced red absorption, Dyes and Pigments 41 (1999), 93-100.

[10] Q.X. Zhen, B.H. Ye, Q.L. Zhang, J.G. Liu, H. Li, L.N. Ji and L. Wang, Synthesis, characterization and the effect of ligand planarity of $\left[\mathrm{Ru}(\mathrm{bpy})_{2} \mathrm{~L}\right]^{2+}$ on DNA binding affinity, J. Inorg. Biochem. 76 (1999), 47-53.

[11] Y.M. Song, J.W. Kang, J. Zhou et al., Study on the fluorescence spectra and electrochemical behavior of $\mathrm{ZnL}_{2}$ and morin with DNA, Spectrochim. Acta A 56 (2000), 2491-2497.

[12] D.G. Dalgleish, G. Fey and W. Kersten, Circular dichroism studies of complexes of the antibiotics daunomycin, nogalamycin, chromomycin and mithramycin with DNA, Biopolymers 13 (1974), 1757-1764.

[13] Y.Y. He, J.Y. An and L.J. Jiang, pH effect on the spectroscopic behavior and photoinduced generation of semiquinone anion radical of hypocrellin B, Dyes and Pigments 41 (1999), 79-87. 


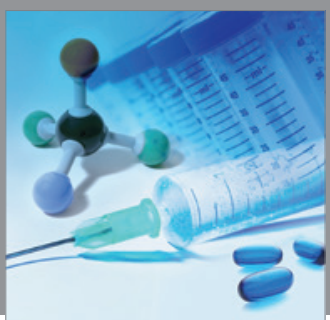

International Journal of

Medicinal Chemistry

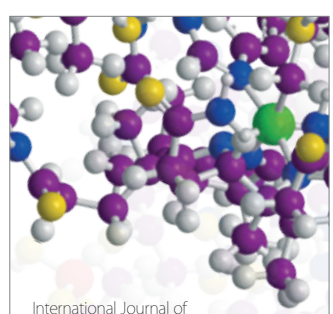

Carbohydrate Chemistry

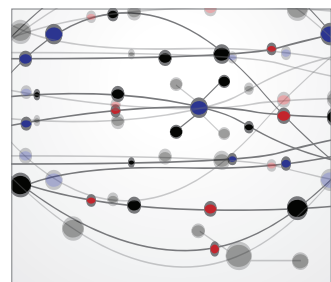

The Scientific World Journal
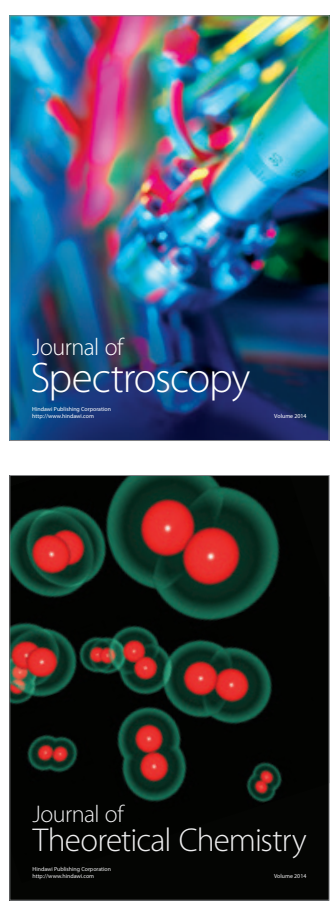
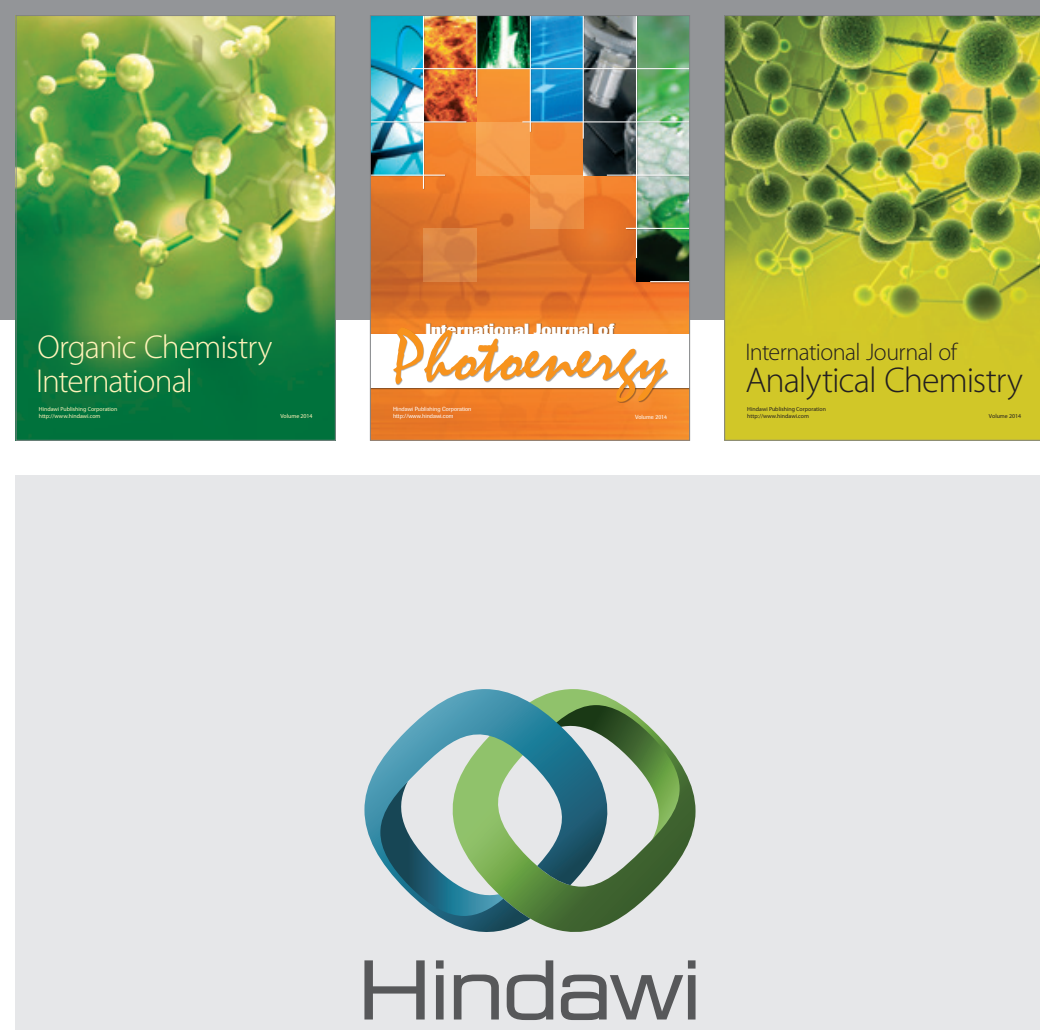

Submit your manuscripts at

http://www.hindawi.com
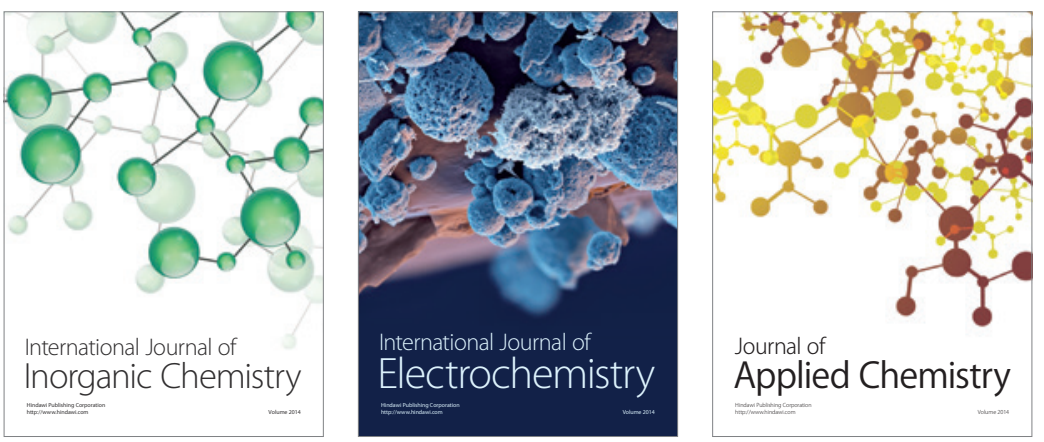

Journal of

Applied Chemistry
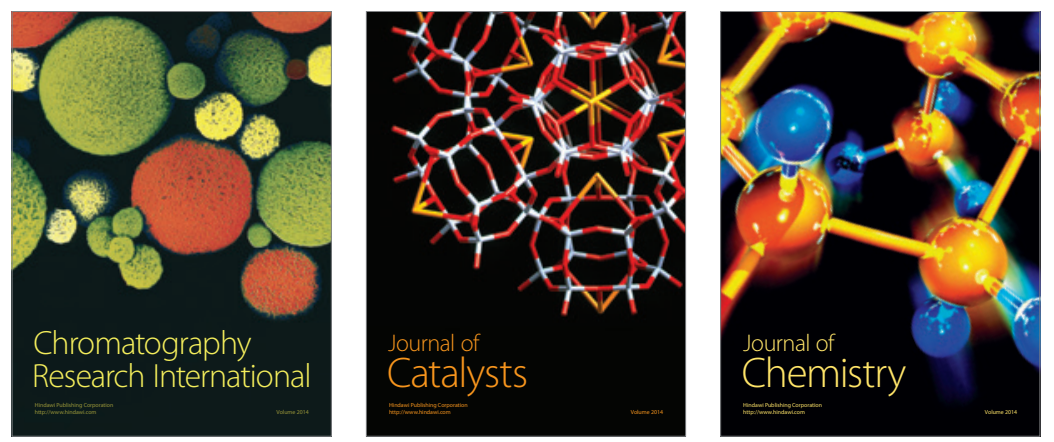
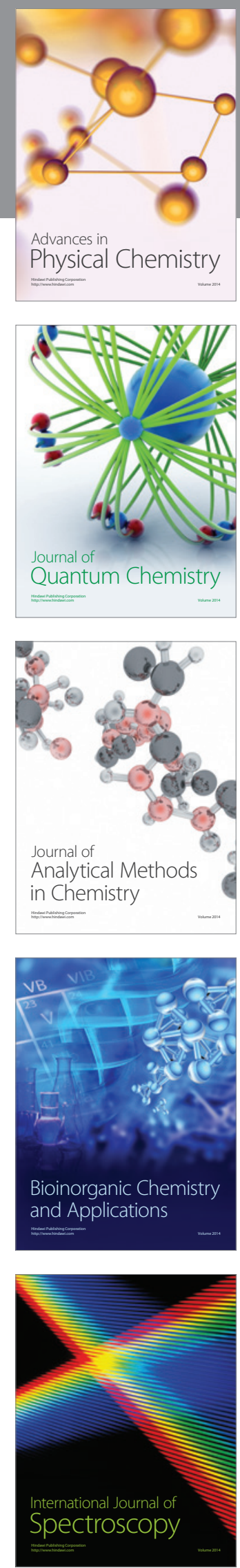